\title{
Device Parameter Changes Caused by Manufacturing Fluctuations of Deep Submicron MOSFET's
}

\author{
Renate Sitte, Student Member, IEEE, Sima Dimitrijev, Member, IEEE, and H. Barry Harrison
}

\begin{abstract}
The effects of typical manufacturing fluctuations upon four electrical device parameters: threshold voltage, transconductance, substrate current and off current have been studied for deep submicron MOSFET's $(0.1 \mu \mathrm{m})$. The analysis reveals that the electrical parameter sensitivity in deep submicron devices differs from micron size devices, making a revision of the validity of conventional semiconductor manufacturing heuristics for future technology mandatory.
\end{abstract}

\section{INTRODUCTION}

$\mathbf{W}$ HILE the effects of processing parameters fluctuations are in the main understood for devices currently produced, little is known about deep submicron devices because little manufacturing experience has yet been accumulated. With down scaling, some physical effects may become more pronounced and dominant over others, changing the effect of manufacturing fluctuations on the device. Several $0.1 \mu \mathrm{m}$ MOSFET's have been designed and manufactured [1]-[4], providing reason to suspect that commercial production at this level may come to fruition in the future. While the published results claim improved device electrical characteristics, these device dimensions raise the important practical question as to whether it will be possible to reproduce these devices in larger numbers. Fluctuations which are found in current manufacturing, and which are sufficiently controlled to be considered as acceptable, may no longer be acceptable for submicron technology. It is the purpose of this paper to identify through the results of analysis, where in future submicron technology the endeavor to minimize device parameter fluctuations are most effective.

To gain insight into the effects of process parameter fluctuations on deep submicron devices a comparative study based on simulation is presented in this paper. The study consisted mainly of three parts: 1) Determination of device parameter fluctuations, based on typical, random fluctuations of processing parameters, using Monte Carlo methods; the device parameters under scrutiny were threshold voltage, transconductance, substrate current, and off-current (the drain current for $V_{G}=0 \mathrm{~V}$ ). 2) Finding for each processing parameter its effective contribution to the device fluctuation. 3) Verification (consolidation) of results.

Manuscript received August 31, 1993; revised June 21, 1994. The review of this paper was arranged by Editor-in-Chief R. P. Jindal.

The authors are with the School of Microelectronic Engineering, Griffith University, Nathan, QLD 4111, Australia.

IEEE Log Number 9405158
TABLE I

\begin{tabular}{|c|c|c|c|c|c|}
\hline Process Parameter & unit & $\begin{array}{l}\text { mean } \\
\text { value }\end{array}$ & std.dev & mean value & std.dev \\
\hline & & \multicolumn{2}{|c|}{ deep submicron } & \multicolumn{2}{|c|}{ micron } \\
\hline Channel Length & $\mu \mathrm{m}$ & 0.1 & 0.06 & 1.5 & 0.15 \\
\hline $\begin{array}{l}\text { Gate Oxide } \\
\text { Thickness }\end{array}$ & $\mathrm{nm}$ & 4.5 & 1.5 & 30 & 3.0 \\
\hline Gate Oxide Charge & $10^{10} \mathrm{~cm}^{-2}$ & 5.0 & 3.0 & 5.0 & 3.0 \\
\hline Substrate Concentr. & $10^{15} \mathrm{~cm}^{-3}$ & 7.0 & 0.7 & 1.0 & 0.1 \\
\hline \multicolumn{6}{|c|}{ Threshold Voltage Adjustment Implant } \\
\hline Species & & \multicolumn{2}{|c|}{ Boron } & \multicolumn{2}{|c|}{ Boron } \\
\hline Dose & $10^{12} \mathrm{~cm}^{-2}$ & 3.0 & 0.03 & 1.1 & 0.011 . \\
\hline Energy & $\mathrm{keV}$ & 15 & 0.15 & 50 & 5 \\
\hline Annealing Temp. & ${ }^{\circ} \mathrm{C}$ & 875 & 0.5 & 1050 & 0.5 \\
\hline Annealing Time & s & 2400 & 1 & 1800 & 1 \\
\hline \multicolumn{6}{|c|}{ Source and Drain Extension Implant } \\
\hline Dose & $10^{14} \mathrm{~cm}^{-2}$ & 4.0 & 0.04 & 25 & 0.025 \\
\hline Energy & $\mathrm{keV}$ & 15 & 0.15 & 50 & 5 \\
\hline Annealing Temp. & ${ }^{\circ} \mathrm{C}$ & 875 & 0.5 & 1050 & 0.5 \\
\hline Annelaing Time & $\mathrm{s}$ & 1800 & 1 & 1800 & 1 \\
\hline
\end{tabular}

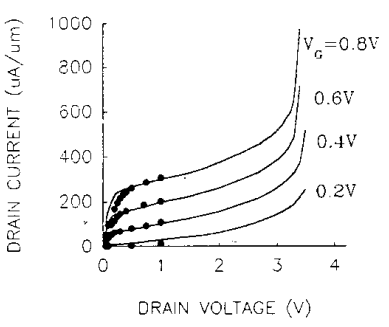

Fig. 1. Output characteristics of $0.1 \mu \mathrm{N}$-channel MOSFET. Symbols: measured data [6], lines: MINIMOS simulation.

\section{CONSTITUENTS OF THE SimUlation EXPERIMENT}

Few deep submicron MOSFET's or circuits have so far been realized [1]-[4]. We have chosen the data published by G. Sai-Halasz et al. [1] for our consideration, namely a 0.1 $\mu \mathrm{m}$ N-channel MOSFET. To be able to make comparisons and detect differences between fluctuations of deep submicron and micron size devices, all simulations were repeated with a typical $1.5 \mu \mathrm{m} \mathrm{N}$-channel device. The fabrication data of these two devices are listed in Table I.

To perform the simulations MINIMOS [5] has been used as the simulation tool. Voltages and currents were obtained with precisions $\pm 0.001 \mathrm{~V}$, and $\pm 10^{-9} \mathrm{~A}$, respectively. While MINIMOS is well known to be accurate for micron size 


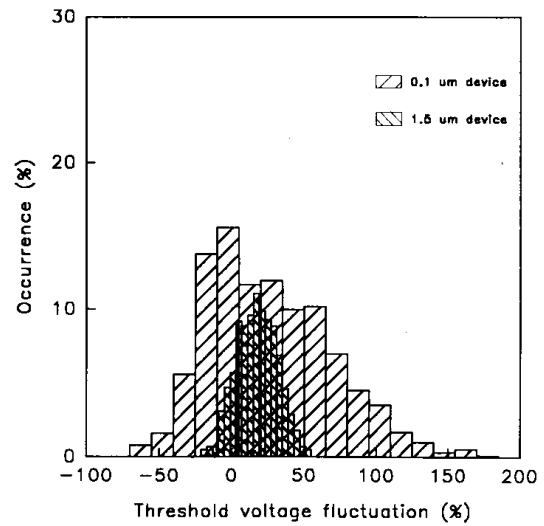

(a)

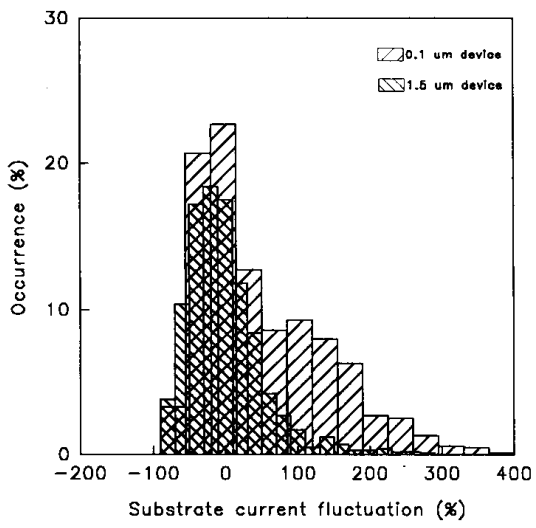

(c)

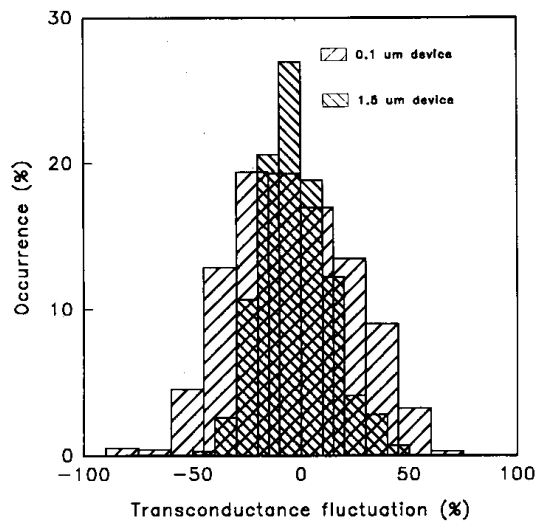

(b)

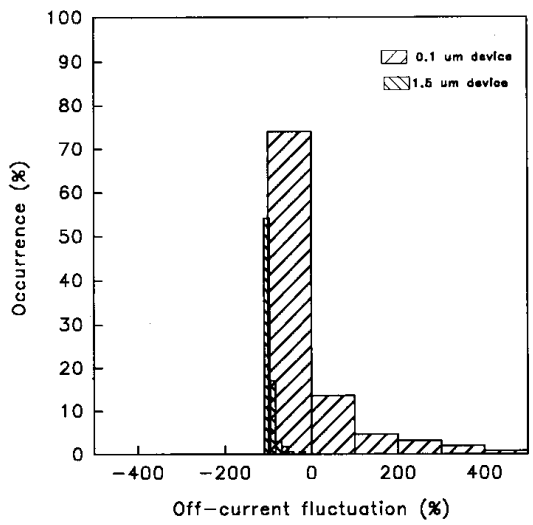

(d)

Fig. 2. Histograms of simulated device parameter fluctuations caused by randomly fluctuating processing parameters: (a) threshold voltage, (b) transconductance, (c) substrate current, and (d) off-current.

devices, its accuracy for small devices was verified previously [6]. To assert that the simulator is sufficiently accurate and consequently an adequate simulator for such a short channel device, the simulation of I-V characteristics have been compared to the measured I-V characteristics. The comparative results are shown in Fig. 1. As can be seen the agreement between the simulation and the actual data suffices to be safely assumed valid for the analyses based on MINIMOS simulations.

\section{General Study of Fluctuation EFFECTS}

To determine the device parameter fluctuations, based on typical, random fluctuations of processing parameters, each device parameter was simulated 1000 times, with data sets generated using Monte Carlo methods. To generate the data sets, each processing parameter was randomly generated from a normal distribution around its recipe value (see Table I), and a standard deviation that is found typically in current manufacturing. The results of these simulations were normalized with respect to their corresponding recipe value, and then the statistical parameters (mean values and standard deviations) were determined for each device parameter. These statistics represent fluctuation phenomena as they may be found in manufacturing, each sample point corresponding to a wafer batch average.

To illustrate any differences, the simulation results are depicted as histograms in Fig. 2. We have found that device parameter fluctuations of the deep submicron device are larger than for the micron size counterparts, although the individual processing parameter fluctuations of the smaller device are equal or proportionally less than those of the large devices. The ratio between their respective standard deviations is at least 2:1 (normalized data).

\section{ANALYTICAL STUdy OF FluCtuAtion EFFECTS}

An analytical study has been performed, to find the most influencing processing parameters, which cause the increased spread of device parameters fluctuations in the small device. A mathematical model which expresses a device parameter variance in terms of the process parameter variances has been used as the starting point in the study. This model was previously applied by Graaff and Klaassen [7] in their study on dependence of circuit parameter variances on device parameter variances. The model applied to the dependence of threshold 


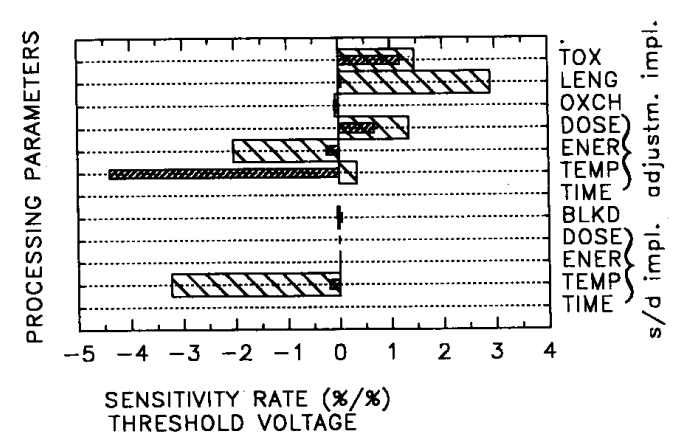

(a)

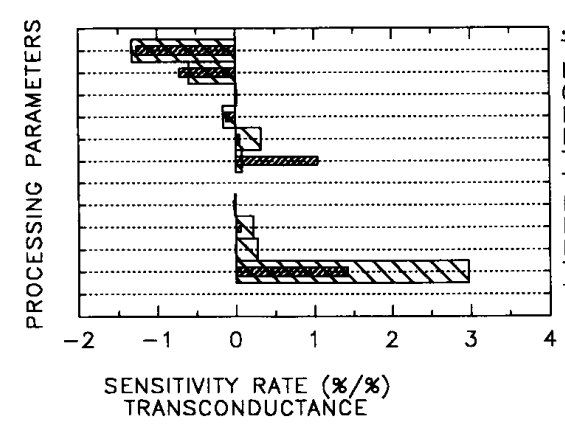

(c)

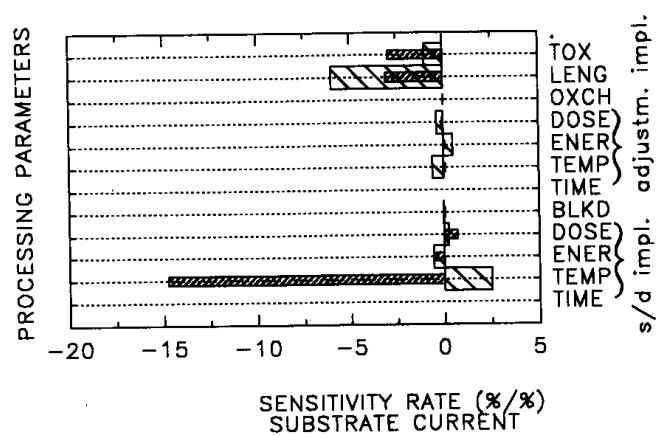

(b)

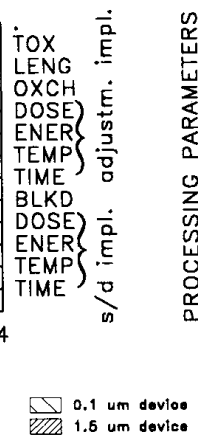

1.5 um device

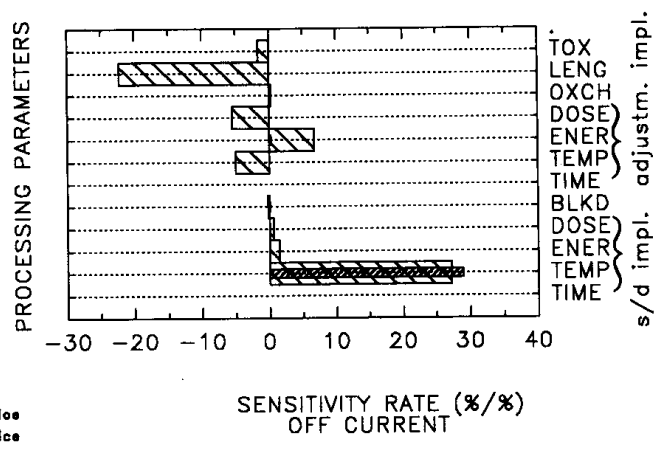

(d)

Fig. 3. Histograms showing the device parameter sensitivity rate to processing fluctuations for (a) threshold voltage, (b) transconductance, (c) substrate current, and (d) off current. All sensitivity data were normalized with respect to the recipe value of the corresponding device parameter.

voltage $\left(V_{\mathrm{th}}\right)$ variance on the process parameter variances has the following form:

$$
\begin{aligned}
\sigma_{\delta V_{t h}}^{2}= & \left(\left.\frac{\partial \delta V_{t h}}{\partial \delta \text { Len }}\right|_{\text {Len }_{\text {spec }}} \sigma_{\delta \text { Len }}\right)^{2}+\left(\left.\frac{\partial \delta V_{\mathrm{th}}}{\partial \delta \text { tox }}\right|_{\text {tox }_{\text {spec }}} \sigma_{\delta \text { tox }}\right)^{2} \\
& +\cdots+\left(\left.\frac{\partial \delta V_{\mathrm{th}}}{\partial \delta \mathrm{t}}\right|_{\mathrm{t}_{\mathrm{spec}}} \sigma_{\delta \mathrm{t}}\right)^{2} .
\end{aligned}
$$

All the quantities in (1) are normalized, which is indicated by the symbol $\delta$, that is $\delta V_{\mathrm{th}}=\left(V_{\mathrm{th}}-\bar{V}_{\mathrm{th}}\right) / \bar{V}_{\mathrm{th}}$. The meaning of the other symbols in (1) is as follows: $\sigma_{\delta V_{\text {th }}}^{2}$ is the variance of the normalized threshold voltage; $\sigma_{\delta L}, \sigma_{\delta \text { tox }}, \ldots, \sigma_{\delta t}$ are the standard deviations of normalized process parameters as listed in Table I; the partial derivatives of the threshold voltage with respect to the process parameters are determined at the points corresponding to the specification (recipe) values of the process parameters. Analogous equations can be written for the other three device parameters under consideration: the transconductance, substrate current, and the off current. It should be noted that generally (1) is a series expansion, including all higher power as well as mixed terms, such as $\frac{\partial^{2} \delta V_{t h}}{\partial \delta L \partial \delta \text { tox }}$. These terms are not explicitly shown in (1) because our subsequent analysis showed that they could be neglected.

This mathematical model provides a basis for the analytical study of the collective influence of the variances of individual process parameters on the variance of a particular device parameter. Equation (1) clearly indicates that it is not enough to know the standard deviations of process parameters, i.e., $\sigma_{L}$, $\sigma_{\text {tox }}, \ldots$, but it is also necessary to determine the derivatives such as $\left.\frac{\partial \delta V_{\mathrm{th}}}{\partial \delta L}\right|_{L_{\text {spec }}}$. These derivatives represent the sensitivity of a particular device parameter on the process parameter fluctuations. To find the sensitivity factors, a sensitivity analysis has been performed [8] as described in the following subsection.

\section{A. Sensitivity Analysis}

This analysis was again based on typical fluctuations found in semiconductor manufacturing, as shown in Table I. It consisted of varying a processing parameter in discrete steps through two standard deviations $(2 \sigma)$ on either side of the recipe value, and simulating the device characteristics. Again this was done for the four device parameters and for each of the listed processing parameters. The resulting device parameter values were linearly fitted, to determine the normalized values of the slopes, which are the sensitivity rates. As a reference for comparisons, the sensitivity rates of a typical micron size device $(1.25 \mu \mathrm{m})$ were also determined, and plotted together with the results of the deep submicron $(0.1 \mu \mathrm{m})$ device in the histograms, which are shown in Fig. 3.

We found that the off current sensitivity due to the channel length was exponential, indicating punch through or avalanche problems [8], [9]. It should be noted that the sensitivity rate for the off-current shown in Fig. 3(d) is a linear approximation, which was done to include this sensitivity rate in our comparative study. 
The sensitivity rates shown in Fig. 3 express percentual fluctuation of a device parameter for any percent of change in a process parameter. Not surprisingly, these histograms show that device parameters are very sensitive to temperature fluctuations (e.g., annealing temperatures for channel and source/drain implants). This does not mean, however, that the temperature contribution to the device parameter fluctuation will be the largest, or even significant. The histograms in Fig. 3 express only sensitivity rates, which are the partial derivatives of a device parameter versus process parameters function, as presented in (1). From this equation, one can see that the sensitivity rates, or the partial derivatives have to be multiplied by the standard deviations of the process parameters, and then squared to obtain a figure which represents a process parameter's contribution to a device parameter fluctuation, or variance. This becomes a fluctuation analysis and it is presented in the following subsection.

\section{B. Fluctuation Analysis}

As mentioned above the effective fluctuation contribution $\varphi$ of a process parameter $p_{i}$ to each of the device parameter's fluctuation is given as squared product of the process parameter's standard deviation and the corresponding sensitivity rate. As an example for the threshold voltage, this expression would be

$$
\varphi=\left(\left.\frac{\partial \delta V_{\mathrm{th}}}{\partial \delta p_{i}}\right|_{p_{i_{\text {spec }}}} \sigma_{p_{i}}\right)^{2} .
$$

To summarize and visualize the effective fluctuations, we have represented them again as histograms shown in Fig. 4. These histograms show the effective fluctuation contribution of each processing parameter to each of the electrical device parameters.

As can be seen from Fig. 4, the effective fluctuations of the device parameters, caused by the process parameter fluctuations, are larger in the deep submicron device in most of the cases. Undoubtedly the two most influencing process parameters are the gate oxide thickness and the channel length. The fluctuations of the gate oxide thickness showed the largest influence on the threshold voltage and the transconductance being four times more effective in the case of threshold voltage and transconductance in the deep submicron device, than in its micron sized counterpart. It has almost equal influence on the substrate current in both device sizes, being slightly larger for the deep submicron device, and finally more than ten times as strong on the off current in the deep submicron device.

The other process parameter that provides an influence is the channel length. While the effect of the channel length fluctuations on the transconductance is surprisingly small for both device sizes under scrutiny, its effect on threshold voltage, substrate current, and off-current is again much larger in the smaller device. As discussed in the previous section, the channel length fluctuations have a marked effect on the off current. This together with the increased sensitivity to threshold voltage adjustment implant energy and oxide charges indicates once more that the off-current, which is generally viewed as a nonessential device parameter, should be carefully investigated when designing deep submicron devices.

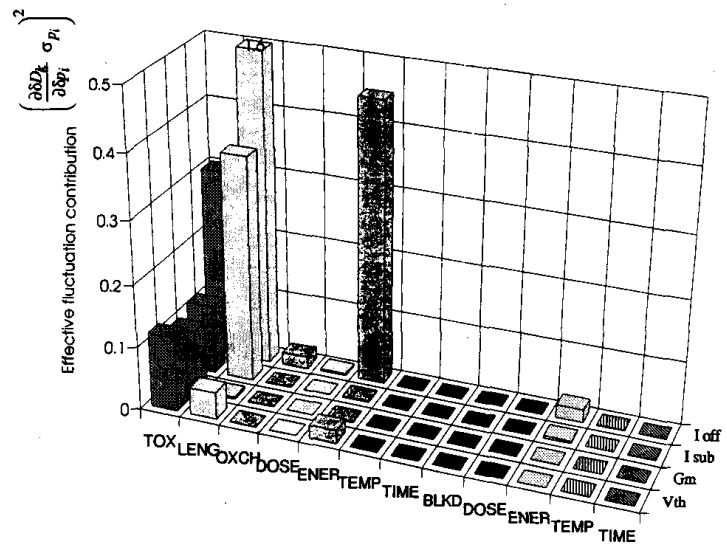

(a)

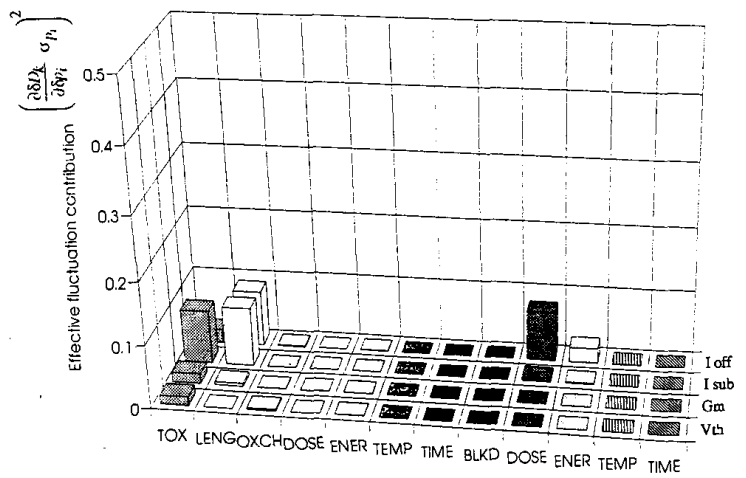

(b)

Fig. 4. Effective contribution of the $i$ th processing parameter $\left(P_{i}\right)$ fluctuations during manufacturing on the resulting electrical device parameter $\left(D_{k}\right)$ fluctuation $\left(\frac{\partial \delta D_{k}}{\partial \delta p_{i}} \partial_{p_{i}}\right)^{2}$ for: a) deep submicron device, b) micron size device. All fluctuation data were normalized $(\delta)$ with respect to the specification value of the corresponding device parameter.

The fluctuations of the threshold voltage adjustment implant, especially the energy, proved to be the third most important cause of device parameter fluctuations. The two most sensitive device parameters were the off-current (with an effective sensitivity up to 500-600 times larger in the deep submicron device than in the micron size device) and the threshold voltage with more than ten fold increase of effective sensitivity in the smaller device.

Fluctuations in the source/drain extension implant were less important, although not negligible, especially in view of off-current fluctuations. The off-current's sensitivity to dose fluctuations is reduced to almost negligible for the smaller device, while the sensitivity to energy is about the same in both devices.

\section{DISCUSSION}

In this study we have determined the sensitivity rate of each processing parameter in an uncorrelated way, independent of any other influence. The reason why we proceeded in this way was not only simplicity, but because wafers are processed in sequential steps, whose manufacturing fluctuations can be assumed independent of each other. In order to verify this 
TABLE II

\begin{tabular}{lcc|cc}
\hline Device Parameter & $\mathbf{0 . 1} \boldsymbol{\mu \mathbf { m }}$ & device & $\mathbf{1 . 5} \boldsymbol{\mu} \mathbf{m}$ & device \\
\hline & \multicolumn{4}{c}{ normalized std. deviation $\%$} \\
\hline Threshold voltage & LHS & RHS & LHS & RHS \\
Transconductance & 27.3 & 42.6 & 13.9 & 13.3 \\
Substrate current & 95 & 32.2 & 15.7 & 14.5 \\
Off-current & 170 & 239 & 53.2 & 43.1 \\
\end{tabular}

independence, we have compared the left hand side of (1), which is the variance of the values of the electrical device parameters from the Monte Carlo simulation, and the right hand side of the same equation, which is the sum of the square values of the processing parameters fluctuation multiplied by the sensitivity rates.

We have verified the independence for the considered four electrical device parameters and the 12 processing parameters as listed above. Again all values were normalized with respect to their specification and recipe value.

We have found that the theorem could be applied with an accuracy better than numerical errors in all the four cases of device parameters. The values obtained for the standard deviations are given in Table II. These values were obtained from the Monte Carlo simulation, left hand side (LHS) of (1) and from the sensitivity analysis, right hand side (RHS) of the same equation.

Comparison of the standard deviations (Table II) provides an insight into the influences of different process parameters. If there were correlations between different process parameters, then neglecting the mixed terms (e.g., $\frac{\partial^{2} \delta V_{\mathrm{th}}}{\partial \delta L \partial \delta \text { tox }}$ ) on RHS of (1) would cause smaller standard deviations compared to their Monte Carlo simulation counterparts (LHS). It can be seen from Table II, that only in the case of substrate current the RHS standard deviations are smaller than their LHS counterparts. The difference however is not significant, keeping in mind that only the current fluctuations towards higher values are undesirable (substrate current fluctuations toward smaller values do not produce any undesirable effects). The discrepancy between both figures in the off-current case cannot be attributed to neglected mixed terms, as the RHS standard deviations are larger than the LHS ones. This discrepancy can only be explained as a numerical error. As one might recall, the gate length exhibited an exponential influence on the off current of the deep submicron device within the range of the typical fluctuation. For the verification of the variance theorem, we have approximated its sensitivity linearly, for the sake of uniformity, introducing a significant error. In the Monte Carlo simulation however, the exponential dependence of the offcurrent on the channel length is inherently used. Further it should be pointed out that the off-current in the micron size device is virtually inexistent, and that even the smallest process parameter fluctuation appears augmented, which explains the relatively large discrepancy of fluctuation figures.

Our results show 1) that the selection of processing parameters chosen for the study are indeed contributing factors to manufacturing fluctuations to the extent as individually determined, 2) that the influences of the processing parameters can be assumed as uncorrelated to each other with reasonable safety, and 3) that only a few process parameters play a major role as affecting through their manufacturing fluctuations the end results of the device parameters.

\section{CONCLUSIONS}

In this study we have identified the main contributors of processing parameter fluctuations during manufacturing to the resulting electrical device parameter fluctuations. The results of our study have revealed that the effects of typical processing fluctuations can be up to 600 times more pronounced in deep submicron devices, than in their micron size counterparts. These huge changes in sensitivity rates indicate that current manufacturing heuristics and efforts for process control need to be revised for future deep submicron technology. The study has also revealed that the effects of processing parameter are largely independent among each other. It is therefore possible to focus efforts to gain better control to those processes which have greater impact in order to reduce their fluctuations.

\section{REFERENCES}

[1] G. A. Sai-Halasz, M. R. Wordeman, D. P. Kern, E. Ganin, S. Rishton, D. S. Zichermann, H. Schmid, M. R. Polcari, H. Y. Ng, P. J. Restle, T. H. P. Chang, and R. B. Dennard, "Design and experimental technology for $0.1-\mu \mathrm{m}$ gate-length low temperature operation MOSFETs," IEEE Electron Device Lett., 1987, vol. 8, pp. 463-466.

[2] M. Aoki, T. Ishii, T. Yoshimura, Y. Kiyota, S. Iijima, T. Yamanaka, T. Kure, K. Ohju, T. Nishida, S. Okazaki, K. Seki, and K. Shimohigashi, "Design and performance of $0.1-\mu \mathrm{m}$ CMOS devices using low impurity channel transistors (LICTs)," IEEE Electron Device Lett., vol. 13, pp. 50-52, Jan. 1992.

[3] M. Iwase, T. Mizuno, M. Takahashi, H. Niiyama, M. Fukumoto, K. Ishida, S. Inaba, Y. Takigami, A. Sanda, A. Toriumi, and $\mathbf{M}$ Yoshimi, "High performance 0.1- $\mu \mathrm{m}$ CMOS devices operating at room temperature," IEEE Electron Device Lett., vol. 14, pp. 51-53, Feb. 1993.

[4] Y. Taur, S. Cohen, S. Wind, T. Lii, C. Hsu, D. Quinlan, C. A. Chang, D. Buchanan, P. Agnello, Y. Mii, C. Reeves, A. Acovoc, and V. Kesan, "Experimental 0.1- $\mu \mathrm{m} p$-channel MOSFET with $p^{+}$-polysilicon gate on 35-A gate oxide," IEEE Electron Device Lett., vol. 14, June 1993.

[5] MINIMOS, Technische Universität Wien, Institut für Mikroelektronik, Austria, 1990.

[6] R. Sitte, S. Dimitrijev, and H. B. Harrison, "The effect of dynamic design processing for yield enhancement in the fabrication of deep-submicron MOSFET's," IEEE Trans. Semicond. Manuf., vol. SM-7, pp. 96-96, Feb. 1984

[7] H. C. de Graaff and F. M. Klaassen, Compact Transistor Modelling for Circuit Design. Wien: Springer-Verlag, 1990.

[8] R. Sitte, S. Dimitrijev, and H. B. Harrison, "Sensitivity of $0.1-\mu \mathrm{m}$ MOSFETs to manufacturing fluctuations," Electronics Lett., vol. 29, no. 15, pp. 1345-1346, July 1993.

[9] R. Sitte, S. Dimitrijev, and H. B. Harrison, "A comparison of parameter sensitivity of deep submicron and micron MOSFET devices on processing parameters," in Simulation of Semiconductor Devices and Processes, S. Selberherr, H. Stippel, and E. Strasser, Eds. New York: Springer-Verlag, vol. 5, 1993, pp. 225-228.

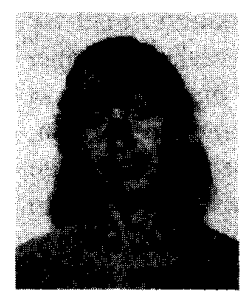

Renate Sitte ( $S^{`} 92$ ) received the engineering degree from the Universidad de los Andes, Venezuela, in 1985, and the M.Phil. in 1992 from Griffith University, Australia.

She has worked as a systems analyst and consultant for systems engineering and operations research in both industry and universities. She joined Griffith University in 1986, where she is presently working toward the Ph.D. in manufacturing fluctuation phenomena and quality control of integrated circuits. Her research interests are in systems engineering. industrial automation, quality control in manufacturing, operations research, and intelligent systems. 


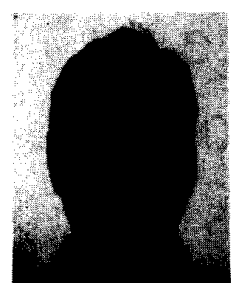

Sima Dimitrijev (S'86-M`88) received the B.Sc., M.Sc., and Ph.D. degrees in electronic engineering from the University of Nis, Yugoslavia, in 1982, 1985 , and 1989 , respectively.

During 1982-1983 he was with the Semiconductor Factory of the Electronic Industry, Nis, where he worked on the development of CMOS technology. From 1983 to 1990 he was with the Faculty of Electronic Engineering at the University of Nis. In 1990 he joined Griffith University, Brisbane, Australia, as a Lecturer of microelectronic engineering. He has published a number of papers on MOS transistor instabilities, IC yield modeling, and MOS transistor modeling.

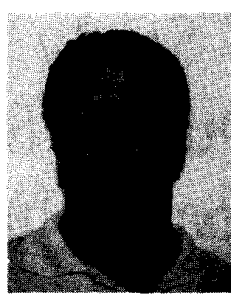

H. Barry Harrison received the B.E. degree in electrical engineering (honors) and the Diploma of Education from the University of Melboume, Australia, in 1965 and 1967, respectively. He received a graduate degree in communication engineering in the Netherlands in 1972 .

He has held various academic and industrial positions around the world, such as Philips Research Laboratory in The Netherlands, and T. J. Watson IBM Research Laboratories and Bell AT\&T in the United States. He served as a Professor at the University of Edinburgh, Scotland, Leuven in Belgium, and Stanford University, Stanford, CA. He is currently Deputy Dean of the Faculty of Science and Technology, and Head of the School of Microelectronic Engineering at Griffith University. 